\title{
Autoavaliação de saúde e doenças crônicas entre enfermeiros de Pelotas/RS
}

\author{
Self-evaluation of health and chronic disease among nurses from Pelotas/RS
}

Autoevaluación de salud y enfermedades crónicas entre enfermeros de Pelotas/RS

Adrize Rutz Porto ${ }^{1}$, Sabrina da Silva Rodrigues², Leandro Rauber Joner ${ }^{3}$, Patrícia Tuerlinckx Noguez ${ }^{4}$, Maira Buss Thofehrn ${ }^{5}$, Daiane Dal Pai ${ }^{6}$

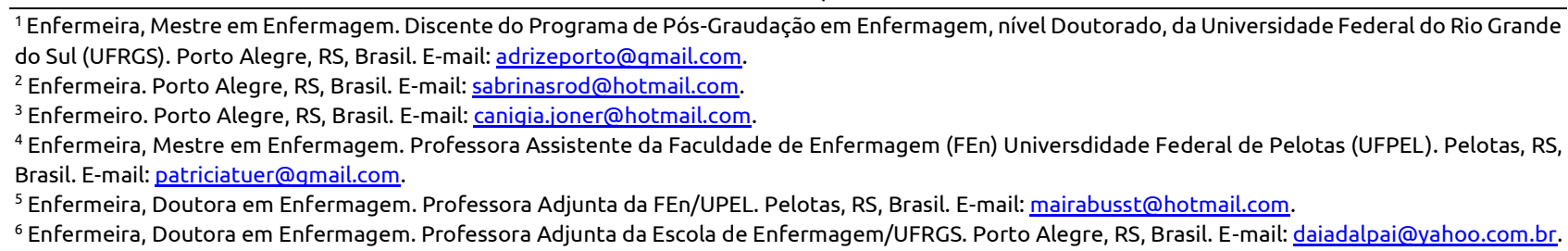

\section{RESUMO}

Descrever a autoavaliação de saúde e a prevalência de doenças crônicas entre enfermeiros. Estudo transversal, realizado com 240 enfermeiros que responderam a questionário estruturado. Fez-se o tratamento dos dados por meio de estatística descritiva e associações com o teste qui-quadrado. A amostra foi composta por enfermeiros, em sua maioria mulheres (90,8\%) adultas jovens (69,6\%), não casadas $(55,4 \%)$ e com filhos $(53,4 \%)$. Na amostra, 35,4\% relataram trabalhar mais de 40 h, $40 \%$ trabalhavam no período noturno, 31,2\% conviviam com doenças crônicas e 37,1\% avaliaram sua saúde de razoável a ruim. Obteve-se associação significativa entre doenças crônicas, idade superior a 39 anos $(p<0,001)$ e autoavaliação de razoável a ruim sobre a saúde $(p<0,001)$. Diante dos achados, emerge a necessidade de investimentos na assistência à saúde dos enfermeiros, principalmente no que tange ao convívio com doenças crônicas, o que, por conseguinte, influenciará na autoavaliação da saúde desses profissionais e no cuidado prestado.

Descritores: Qualidade de Vida; Saúde do Trabalhador; Enfermagem.

\section{ABSTRACT}

To describe the self-evaluation of health and the prevalence of chronic diseases among nurses. This cross-sectional study was performed with 240 nurses, who answered a structured questionnaire. Data treatment performed using descriptive statistics and association with the chi-square test. The sample consisted of nurses, mostly women (90.8\%), young adults (69.6\%), unmarried (55.4\%) and with children (53.4\%). In the sample, 35.4\% reported working more that $40 \mathrm{~h}, 40 \%$ worked the night shift, $31.2 \%$ had chronic diseases, and $37.1 \%$ evaluated their health as being between average and bad. A significant association was obtained between chronic diseases and age above 39 years $(p<0.001)$, and a self-evaluation of health between average and bad $(p<0.001)$. The findings reveal it is necessary to invest in nurses' health care, mainly regarding their living with chronic diseases, which, in turn, will affect the self-evaluation that these professionals make of their own health and of the care that is delivered.

Descriptors: Quality of Life; Occupational Health; Nursing.

\section{RESUMEN}

Describir la autoevaluación de salud y la prevalencia de enfermedades crónicas entre enfermeros. Estudio transversal, realizado con 240 enfermeros que respondieron cuestionario estructurado. Datos tratados por estadística descriptiva y asociaciones con test de Chi-cuadrado. Muestra compuesta por enfermeros, en su mayoría mujeres (90,8\%), adultas jóvenes (69,6\%), no casadas $(55,4 \%)$ y con hijos $(53,4 \%)$. En la muestra, $35,4 \%$ informó trabajar más de 40 hs, $40 \%$ trabajaba en horario nocturno, 31,2\% convivía con enfermedades crónicas y 37,1\% evaluaron su salud de razonable a mala. Se obtuvo asociación significativa entre enfermedades crónicas y edad superior a 39 años $(p<0,001)$ y autoevaluación de salud de razonable a mala $(p<0,001)$. Ante tales hallazgos, surge la necesidad de inversiones en atención de salud de los enfermeros, particularmente en lo atinente a la convivencia con enfermedades crónicas, lo cual, por consiguiente, influirá en la autoevaluación de salud de tales profesionales, y en los cuidados brindados.

Descriptores: Calidad de Vida; Salud Laboral; Enfermería. 


\section{INTRODUÇÃO}

A Organização Mundial da Saúde considera qualidade de vida (QV) como a visão do ser humano frente a sua vida, cultura, sistema de valores, além da relação com seus objetivos, expectativas e preocupações ${ }^{(1)}$. Assim, a QV de um indivíduo está associada ao que o próprio espera do mundo que está a sua volta e ao que ele faz para atingir suas propostas.

Ao refletir sobre os aspectos que permeiam a vida de uma pessoa, o trabalho pode ser compreendido como revelador da essência de cada ser humano, atuando como um agente de transformação, com o qual estão implicadas a sobrevivência e realização pessoal(2). Dessa forma, o trabalho passa a interferir diretamente na QV das pessoas, visto que ele pode potencializar a saúde ou o adoecimento, com danos sobre a integridade física e/ou psicológica.

Se antigamente o nexo causal do labor com a saúde dos indivíduos era estabelecido pela relação entre a exposição ao agente ou tarefa nociva e o aparecimento da doença, na atualidade, sabe-se que o expressivo aumento das doenças crônicas e degenerativas não tem tido relação direta com um fator presente na labuta, mas encontra-se implicada na intensidade e tempo de sujeição às condições de trabalho ${ }^{(3)}$.

Os problemas de saúde entre os profissionais da enfermagem têm gerado importantes perdas para o trabalhador, assim como para as instituições de saúde. Além do absenteísmo, é frequente encontrar trabalhadores com esgotamento, deprimidos ou ansiosos, porém as consequências do desempenho prejudicado e da baixa produtividade ainda são pouco conhecidas no contexto da enfermagem. Mesmo em processo de adoecimento, muitos trabalhadores permanecem em atividade, fenômeno que pode estar relacionado ao grau de motivação, engajamento ou satisfação com o trabalho(4). Além disso, muitas vezes, as pessoas com doenças crônicas não se percebem doentes, sobretudo por não apresentarem sintomas, não serem, ainda, portadores de incapacidades ou não possuírem, em dado momento, outras comorbidades ${ }^{(5)}$.

Ainda, o estresse crônico tem sido um agravo observado no trabalho da enfermagem. Denominado como Síndrome de Burnout, a resposta prolongada a estressores emocionais e interpessoais é uma síndrome frequentemente observada em atividades laborais que exigem interações intensas e constantes com o público.
Diante disso, os enfermeiros têm sido profissionais especialmente expostos ao agravo, em razão das altas demandas físicas e emocionais exigidas no trabalho(6).

Os trabalhadores de enfermagem ao conviverem diariamente com exigências cognitivas e psíquicas no desempenho das inúmeras e variadas funções que envolvem o processo de cuidar, precisam, para tal, contar com um ambiente de trabalho que seja acolhedor e organizado. Dessa forma, entende-se que o espaço laboral e as relações sociais que nele se constituem, contribuem para o bem-estar e a saúde dos enfermeiros.

O trabalho em saúde sofre, ainda, influências do contexto político e econômico, uma vez que tanto as forças micro quanto macroestruturais acabam interferindo nas relações sócio-laborais. As condições de trabalho, por vezes, não favorecem o desenvolvimento pleno das atividades previstas, o que também contribui para a desvalia do esforço humano, de maneira a prejudicar os trabalhadores nos demais aspectos de suas vidas, pois o trabalho, a saúde e a vida são inerentes um ao outro. No que se refere à gestão do trabalho, ressaltam-se algumas questões que se relacionam diretamente à QV, como as tecnologias e seu impacto para a saúde, o bem-estar no trabalho com associação direta ao nível salarial, a criatividade e a autonomia do trabalhador para o exercício do protagonismo nas condições, ambientes e organização do processo de trabalho ${ }^{(7)}$.

Nessa perspectiva, entende-se que a percepção do trabalhador sobre os benefícios do seu esforço laboral é fator condicionante do seu comportamento, bem como a autoavaliação da saúde resulta das potencialidades ou limitações impostas pela condição biopsíquica do indivíduo a sua QV. Nesta direção, estudo constatou associação entre a autopercepção insatisfatória dos enfermeiros com o seu estado de saúde e a baixa $Q{ }^{(8)}$.

A autoavaliação de saúde tem sido considerada por estudiosos $^{(5,9)}$ relevante preditor de morbidade, a qual se baseia em critérios subjetivos e objetivos, sendo influenciada por fatores como sexo, idade, classe social e presença de doenças crônicas. Diante do exposto, o objetivo do presente estudo foi descrever o perfil sociodemográfico e a autoavaliação de saúde dos enfermeiros, partindo-se do pressuposto de que esses dados fornecem subsídios importantes para a análise da QV dos profissionais enfermeiros. Este trabalho integra a pesquisa "Qualidade de Vida no Trabalho dos 
Enfermeiros de Pelotas" e vincula-se ao Núcleo de Estudos em Práticas de Saúde e Enfermagem (NEPEn) da Faculdade de Enfermagem da Universidade Federal de Pelotas (UFPel), RS.

\section{MÉTODO}

Trata-se de um estudo de delineamento transversal realizado com enfermeiros do município de Pelotas, Rio Grande do Sul (RS), no período de agosto de 2009 a abril de 2010. A amostra foi constituída por 240 profissionais $(\mathrm{N}=240)$, selecionados aleatoriamente a partir de uma listagem de 320 dos enfermeiros com vínculo empregatício, fornecida pela subseção do Conselho Regional de Enfermagem (COREN) no município.

Os enfermeiros incluídos foram abordados em seu local de trabalho, ou seja, em hospitais, unidades básicas de saúde ou instituições de ensino, conforme preferência de horário e disponibilidade. A coleta de dados foi realizada, por acadêmicos de enfermagem, previamente capacitados. Foram excluídos do estudo, enfermeiros em férias, período de afastamento, por atestado ou licença saúde. Não houve recusas à participação no estudo.

A amostra foi calculada admitindo-se um erro amostral máximo de $5 \%$ com um nível de confiança de $95 \%$. Os enfermeiros selecionados responderam ao instrumento de coleta dos dados avaliado por teste piloto, composto por 15 questões fechadas, elaborada pelos próprios pesquisadores. Foram avaliados aspectos sociodemográficos e laborais, como sexo, idade, estado civil, filhos ou dependentes, crença espiritual, tempo de formação, pós-graduação, tempo de atuação, turno de trabalho, carga horária semanal, áreas de trabalho, vínculo empregatício e tempo para o lazer. Com base na relevância da autoavaliação da própria saúde do indivíduo face aos aspectos sociodemográficos ${ }^{(5,9)}$, solicitou-se que os respondentes autoavaliassem a sua saúde com base nos critérios: ruim, razoável, boa ou muito boa. Por fim, os participantes responderam sobre a presença ou ausência de doenças crônicas, sendo possível descrever, por extenso, ao lado da questão, de que doença se tratava.

Após a coleta dos dados, realizou-se a dupla digitação dos dados no programa Microsoft Excel 2007. A análise dos dados efetuou-se por meio de estatística descritiva no software SPSS, versão 18.0 (Statistical Package for the Social Sciences).

Os resultados foram expressos com números absolutos, incluindo-se seus percentuais e, para análise do nível de associação, utilizou-se o teste qui-quadrado. Foram consideradas diferenças estatisticamente significativas as associações com $p<0,05$.

A todos os participantes foi explicado o Termo de Consentimento Livre e Esclarecido antes de ser assinado em duas vias, autorizando a participação no estudo. 0 projeto de pesquisa foi previamente aprovado pelo Comitê de Ética e Pesquisa da Faculdade de Enfermagem da Universidade Federal de Pelotas, sob o número 20/2009.

\section{RESULTADOS}

A aplicação dos questionários permitiu conhecer a prevalência de enfermeiros do sexo feminino na amostra (90,8\%), com idade inferior a 39 anos $(69,6 \%)$, com um ou mais filhos $(53,4 \%)$ e com mais de cinco anos de formação em enfermagem (65,4\%). A Tabela 1 apresenta esses e outros dados que caracterizam o perfil sociodemográfico e laboral dos enfermeiros estudados, bem como a sua autoavaliação de saúde. 
Tabela 1: Perfil sociodemográfico e autoavaliação de saúde dos enfermeiros de Pelotas/RS, 2010.

\begin{tabular}{|c|c|c|}
\hline Indicadores & $\mathbf{N}$ & $\%$ \\
\hline \multicolumn{3}{|l|}{ Sexo $(N=240)$} \\
\hline Feminino & 218 & 90,8 \\
\hline Masculino & 22 & 9,2 \\
\hline \multicolumn{3}{|l|}{ Idade $(\mathrm{N}=240)$} \\
\hline $20-39$ anos & 167 & 69,6 \\
\hline Mais de 39 anos & 73 & 30,4 \\
\hline \multicolumn{3}{|l|}{ Estado Civil ( $\mathrm{N}=239 *)$} \\
\hline Casado & 106 & 44,2 \\
\hline Outro & 133 & 55,4 \\
\hline \multicolumn{3}{|l|}{ Filhos ou dependentes $(\mathrm{N}=237$ *) } \\
\hline Não & 109 & 45,4 \\
\hline Um ou mais & 128 & 53,4 \\
\hline \multicolumn{3}{|l|}{ Crença Espiritual ( $\mathrm{N}=\mathbf{2 4 0}$ ) } \\
\hline Não & 21 & 8,8 \\
\hline Sim & 219 & 91,2 \\
\hline \multicolumn{3}{|l|}{ Tempo de formação ( $N=239 *)$} \\
\hline Menos de 5 anos & 82 & 34,2 \\
\hline Mais de 5 anos & 157 & 65,4 \\
\hline \multicolumn{3}{|l|}{ Pós-Graduação ( $\mathrm{N}=237$ *) } \\
\hline Não & 72 & 30,0 \\
\hline Sim & 165 & 68,8 \\
\hline \multicolumn{3}{|l|}{ Tempo de atuação ( $\mathrm{N}=\mathbf{2 4 0}$ ) } \\
\hline Menos de 5 anos & 81 & 33,8 \\
\hline Mais de 5 anos & 159 & 66,2 \\
\hline \multicolumn{3}{|l|}{ Turno de trabalho ( $\mathrm{N}=236 *)$} \\
\hline Diurno & 140 & 58,3 \\
\hline Noturno & 96 & 40,0 \\
\hline \multicolumn{3}{|l|}{ Carga Horária Semanal (N=238*) } \\
\hline Até 40h & 133 & 55,4 \\
\hline Mais de 40h & 85 & 35,4 \\
\hline \multicolumn{3}{|l|}{ Áreas de trabalho $(\mathrm{N}=240)$} \\
\hline Assistência & 203 & 84,6 \\
\hline Ensino & 37 & 15,4 \\
\hline \multicolumn{3}{|l|}{ Vinculo empregatício ( $\mathrm{N}=240$ ) } \\
\hline Apenas um local & 183 & 76,2 \\
\hline Dois locais ou mais & 57 & 23,8 \\
\hline \multicolumn{3}{|l|}{ Tempo para o lazer $(\mathrm{N}=240)$} \\
\hline Sim & 147 & 61,2 \\
\hline Não & 93 & 38,8 \\
\hline \multicolumn{3}{|l|}{ Doenças Crônicas ( $\mathrm{N}=\mathbf{2 4 0}$ ) } \\
\hline Não & 165 & 68,8 \\
\hline Sim & 75 & 31,2 \\
\hline \multicolumn{3}{|l|}{ Autoavaliação de Saúde ( $N=240)$} \\
\hline Boa a muito boa & 151 & 62,9 \\
\hline Ruim a razoável & 89 & 37,1 \\
\hline
\end{tabular}

*N<240 por respostas ignoradas

Dessa forma, foi possível constatar que $31,2 \%$ dos enfermeiros afirmaram alguma doença crônica, dentre as quais foram citadas a hipertensão arterial, a diabetes mellitus, os transtornos depressivos e as doenças osteomusculares. Na autoavaliação de saúde, 37,1\% dos enfermeiros classificaram sua saúde como ruim a razoável.

A Tabela 2 ainda apresenta a distribuição dos participantes do estudo quanto ao convívio com pelo menos um tipo de doença crônica. 
Tabela 2: Distribuição de Doenças Crônicas em Enfermeiros de Pelotas segundo variáveis sociodemográficas e autoavaliação de saúde. Pelotas/RS, 2010.

\begin{tabular}{|c|c|c|c|}
\hline \multirow{2}{*}{ Variáveis } & \multicolumn{2}{|c|}{ Doenças Crônicas } & \multirow{2}{*}{$\mathbf{P}$} \\
\hline & Sim N (\%) & Não N (\%) & \\
\hline \multicolumn{4}{|l|}{ Sexo } \\
\hline Feminino & $69(31,7)$ & $149(68,3)$ & 0,811 \\
\hline Masculino & $06(27,3)$ & $16(72,7)$ & \\
\hline \multicolumn{4}{|l|}{ Idade } \\
\hline 20-39 anos & $40(24)$ & $127(76)$ & $<0,001$ \\
\hline Mais de 39 anos & $35(47,9)$ & $38(52,1)$ & \\
\hline \multicolumn{4}{|l|}{ Estado Civil } \\
\hline Casado & $32(30,2)$ & $74(69,8)$ & 0,888 \\
\hline Outro & $42(31,6)$ & $91(68,4)$ & \\
\hline \multicolumn{4}{|l|}{ Filhos ou dependentes } \\
\hline Não & $26(23,9)$ & $83(76,1)$ & 0,018 \\
\hline Sim & $49(38,3)$ & $79(61,7)$ & \\
\hline \multicolumn{4}{|l|}{ Crença espiritual } \\
\hline Não & $07(33,3)$ & $14(66,7)$ & 0,809 \\
\hline Sim & $68(31,1)$ & $151(68,9)$ & \\
\hline \multicolumn{4}{|l|}{ Tempo de formação } \\
\hline Menos de 5 anos & $10(12,2)$ & $72(87,8)$ & $<0,001$ \\
\hline Mais de 5 anos & $64(40,8)$ & $93(59,2)$ & \\
\hline \multicolumn{4}{|l|}{ Pós-Graduação } \\
\hline Não & $16(22,2)$ & $56(77,8)$ & 0,048 \\
\hline Sim & $59(35,8)$ & $106(64,2)$ & \\
\hline \multicolumn{4}{|l|}{ Turno de trabalho } \\
\hline Diurno & $42(30)$ & $98(70)$ & 0,669 \\
\hline Noturno & $32(33,3)$ & $64(66,7)$ & \\
\hline \multicolumn{4}{|l|}{ Tempo de atuação } \\
\hline Menos de 5 anos & $11(13,6)$ & $70(86,4)$ & $<0,001$ \\
\hline Mais de 5 anos & $64(40,3)$ & $95(59,7)$ & \\
\hline \multicolumn{4}{|l|}{ Carga horária semanal } \\
\hline Até 40h & $40(30,1)$ & $93(69,9)$ & 1 \\
\hline Mais de 40h & $25(29,4)$ & $60(70,6)$ & \\
\hline \multicolumn{4}{|l|}{ Áreas de trabalho } \\
\hline Assistência & $63(31)$ & $140(69)$ & 0,849 \\
\hline Ensino & $12(32,4)$ & $25(67,6)$ & \\
\hline \multicolumn{4}{|l|}{ Vínculo empregatício } \\
\hline Apenas um local & $55(30,1)$ & $128(69,9)$ & 0,514 \\
\hline Dois locais ou mais & $20(35,1)$ & $37(64,9)$ & \\
\hline \multicolumn{4}{|l|}{ Tempo para o lazer } \\
\hline Sim & $48(32,7)$ & $99(67,3)$ & 0,571 \\
\hline Não & $27(29)$ & $66(71)$ & \\
\hline \multicolumn{4}{|l|}{ Autoavaliação de saúde } \\
\hline Boa a muito boa & $32(21,2)$ & $119(78,8)$ & $<0,001$ \\
\hline Ruim a razoável & $43(48,3)$ & $46(51,7)$ & \\
\hline
\end{tabular}

Revela-se, assim, associação estatisticamente significativa entre as doenças crônicas e as variáveis: possuir filhos $(p=0,018)$, qualificação profissional na pósgraduação $(p=0,048)$, maior tempo de atuação $(p<0,001)$, maior tempo de formação $(p<0,001)$ idade superior a 39 anos $(p<0,001)$ e autoavaliação de saúde ruim a razoável $(p<0,001)$.

\section{DISCUSSÃO}

No que se refere às características sociodemográficas da categoria profissional pesquisada, identificou-se uma população predominantemente do sexo feminino, adulto jovem e não casada, o que se assemelha a outros trabalhos realizados com enfermeiros ${ }^{(8,10)}$.

A prevalência de enfermeiros do sexo feminino demonstra uma tendência nacional(11) e internacional(12) da profissão. Assim como, o predomínio de adultas jovens não casadas pode estar evidente na relativa aceitabilidade do mercado de trabalho, por tais características garantirem maior disponibilidade para o serviço, quando estas não possuem obrigações familiares além do trabalho, como cuidar das tarefas domésticas, marido e filhos. 
Ademais, as mulheres que são casadas, apresentam taxas mais baixas de inserção no mercado de trabalho, seja por discriminação ou por encargos familiares ou domésticos ${ }^{(13)}$. Essa constatação é corroborada por uma investigação que identificou $79,1 \%$ dos trabalhadores de enfermagem que revelaram que as atividades mais praticadas fora do trabalho eram os afazeres domésticos ${ }^{(14)}$.

Por outro lado, outra pesquisa aponta que os enfermeiros que possuem companheiro apresentaram melhores escores de QV se comparados aos que não possuem ${ }^{(8)}$. Todavia, chama à atenção o fato de que, apesar de não casados, prevalecem os enfermeiros com um ou mais filhos/dependentes, perfazendo $53,4 \%$ da amostra, um baixo número se comparado com outro estudo realizado no mesmo estado (RS), onde $80,9 \%$ dos enfermeiros possuem um ou mais filhos/dependentes ${ }^{(15)}$. Isso pode ser explicado pelas intensas demandas da vida contemporânea, em que os enfermeiros assumem duplas ou triplas jornadas de trabalho, principalmente, em função da baixa remuneração salarial dos enfermeiros no município pesquisado.

Outro aspecto levantado foi a crença espiritual, o qual se mostrou presente em $91,2 \%$ dos enfermeiros participantes da pesquisa, podendo ratificar a importância da espiritualidade no enfrentamento das dificuldades impostas pelo processo de trabalho. Em relação à crença religiosa, investigação apontou que a maioria dos enfermeiros pertencia à religião católica $(69,57 \%)$ e $17,39 \%$ deles afirmaram não ter religião ${ }^{(11)}$.

No que diz respeito ao tempo de formação acadêmica e de atuação profissional foi identificado que cerca de $60 \%$ dos entrevistados possuem mais de cinco anos de graduação e atuação, como em outros trabalhos ${ }^{(11,16)}$, o que pode levar à identificação da prevalência de um perfil de enfermeiro com razoável tempo de experiência, atuando nas instituições de Pelotas. Ainda pode-se constatar que $68,8 \%$ dos enfermeiros são pós-graduados, tal como se apresenta em outros estudos; os quais demonstraram a busca por aperfeiçoamento após a graduação ${ }^{(8,10)}$.

Ainda em relação ao percentual de enfermeiros com formação de pós-graduação (68,8\%), pode-se relacionar à maior seletividade do mercado de trabalho e à esperança de aumento salarial ${ }^{(8)}$. Outra pesquisa verificou que existe tendência de aumentar a proporção de trabalhadores capacitados para o atendimento com qualidade na medida em que melhora o nível de escolaridade ${ }^{(17)}$.

Ao identificar os turnos de trabalho e a carga horária semanal de atuação dos enfermeiros pelotenses, constatou-se o predomínio dos turnos manhã e/ou tarde, com uma carga horária de até 40 horas semanais. Essa questão está relacionada ao fato de que alguns serviços, como os da atenção básica e a maioria das escolas de enfermagem, funcionarem somente no turno diurno ou, ainda pode estar associado ao fato de os hospitais empregarem uma maior quantidade de enfermeiros durante o dia, devido ao número expressivo de tarefas a serem desenvolvidas no período diurno(18). Um trabalho com enfermeiros apresentou a carga horária laboral com média de 37,5 horas por semana de 235 enfermeiros ${ }^{(11)}$.

Nesse sentido, as longas jornadas de trabalho de 12 horas e 24 horas, plantões noturnos, e o multiemprego são exemplos de algumas modalidades de trabalho que têm sido associadas aos distúrbios musculoesqueléticos, à obesidade e aos hábitos de vida pouco saudáveis, tais como: dormir menos de seis horas por dia e o maior consumo de bebidas alcoólicas e cigarro, além de que estes fatores aumentam a chance de desenvolvimento de diversos problemas de saúde, em especial, aos problemas cardiovasculares $^{(4)}$.

Pode-se constatar que a maior parte dos enfermeiros se concentra na assistência, no qual 76,2\% trabalham em apenas um local, diferentemente de outro estudo, em que foi apontada a presença de mais de um vínculo empregatício dos enfermeiros (53\%) no Rio de Janeiro ${ }^{(16)}$. Cabe destacar que as múltiplas jornadas de trabalho e baixa remuneração provocam desgaste da saúde dos profissionais $^{(19)}$.

Sabe-se, no entanto, que essa condição de elevada carga horária semanal de trabalho e pouco tempo para lazer coloca o profissional à margem da sobrecarga de tarefas e desgaste causado pelo estresse, gerando sinais e sintomas como taquicardia, falta de apetite, calafrios, ansiedade e dores articulares ${ }^{(20)}$.

O estresse pode levar a Síndrome de Burnout que é a exaustão emocional, caracterizada pela carência de energia e entusiasmo em razão do esgotamento dos recursos pessoais(14) e a baixa realização no trabalho, caracterizada pela tendência do trabalhador autoavaliar sua saúde de forma negativa(21).

Relativo ao tempo para o lazer, $61,2 \%$ dos enfermeiros afirmou o ter, sendo que um estudo 
realizado com profissionais de enfermagem pormenoriza que $87 \%$ dos sujeitos têm um momento de lazer e $26,48 \%$ praticam algum exercício físico ${ }^{(22) .} \mathrm{Em}$ outro levantamento, constatou-se que $37,2 \%$ dos trabalhadores praticam atividades físicas ${ }^{(14)}$. Uma pesquisa refere que os enfermeiros, os quais estão no início do tratamento de doenças, realizam mais atividades de lazer, sendo estas mais de entretenimento (44,44\%), seguido por atividades físicas $(30,87 \%)$ e viagens (24,69\%), porém com a evolução do tempo de diagnóstico tendem a reduzir em até $50 \%$ estas atividades ${ }^{(11)}$.

Nesta investigação $31,2 \%$ dos enfermeiros afirmou conviver com doenças, tais como: hipertensão arterial, diabetes, transtornos depressivos e doenças osteomusculares. Em outro estudo, 19,9\% dos profissionais de enfermagem eram hipertensos ${ }^{(22)}$ e noutro, a maioria dos enfermeiros possuíam doenças cardiovasculares ${ }^{(10)}$. Ainda, uma pesquisa apontou que a maioria dos enfermeiros relatou como fator de risco a hipertensão arterial $(21,10 \%)$, seguido dos antecedentes familiares de doenças crônicas $(18,90 \%)$ e obesidade $(14,40 \%)^{(11)}$.

A equipe de enfermagem vivencia nítida sobrecarga de trabalho, por isso tem sido considerada a mais afetada por problemas de saúde entre os profissionais da saúde, principalmente, pelas doenças do sistema osteomuscular e do tecido conjuntivo na região dos membros superiores, apresentando dor, entre as queixas mais comuns, além da parestesia e diminuição da força muscular. Cabe ressaltar que, muitas vezes, nesse tipo de doença, a invisibilidade dos sinais e a subjetividade das queixas, agregado ao pouco conhecimento dos trabalhadores sobre ela, levam estes a não se importar com os sintomas iniciais e, assim, não procurarem assistência médica no tempo devido(23).

Não obstante, ainda acontece de profissionais preferirem suportar o sofrimento, durante as jornadas de trabalho, pelo medo da demissão ou receio da discriminação que venham a lidar no retorno às atividades laborais, mesmo que essa conduta possa ocasionar uma piora no processo de adoecimento ${ }^{(24)}$. Além disso, o relato de ausência de doenças por enfermeiros em $68,8 \%$ vai ao encontro de outra investigação, a qual revelou $66,7 \%$ dos indivíduos na mesma condição(8).

Quanto à autopercepção de saúde, 62,9\% dos enfermeiros consideram sua saúde "boa a muito boa". Em um trabalho foi identificado $79,8 \%$ de profissionais nessa condição(12) e em outro inquérito, 92\% dos indivíduos caracterizou sua saúde como sendo "boa" ou "muito boa"(8). Entretanto, uma pesquisa com 1.307 profissionais de enfermagem teve como desfecho o estado de saúde auto-referido como "boa" por $24,8 \%$ dos entrevistados, "regular" por 55,6\% participantes, "ruim" por 18,3\% trabalhadores e "péssima" por apenas $1,2 \%$ dos profissionais $^{(25)}$.

Desta forma, o fato de $37,1 \%$ dos enfermeiros considerarem a condição de sua saúde de "razoável a ruim" pode estar relacionada à falta de qualidade de vida dos mesmos(8), isso estimula os pesquisadores a aprofundar e a dar continuidade aos estudos com tal temática, bem como aprofundar relações entre os agravos psíquicos e físicos e a QV.

Frente às respostas dadas pelos participantes, foi encontrada associação estatisticamente significativa entre as doenças crônicas e possuir filhos, resultado similar a outro estudo ${ }^{(25)}$. Sobre esses dados, infere-se que as enfermeiras, além de cumprirem o seu papel de profissional de saúde, são encarregadas pelo cuidado dos seus membros da família, especialmente, a socialização e criação dos filhos. Trata-se, na maioria das vezes, de profissionais com duplas ou triplas jornadas de trabalho por incluírem à responsabilidade profissional, a administração dos processos familiares, a execução de tarefas relacionadas com a alimentação e higiene da sua casa e a regulação dos afetos do casal e da família, etc ${ }^{(25)}$.

Os dados ainda apontam associação significativa entre as variáveis tempo de atuação, tempo de formação e a maior idade com a presença de doenças crônicas. Isso requer considerar, o acréscimo dos efeitos do desgaste profissional sobre a vulnerabilidade ao adoecimento.

Em outra pesquisa com enfermeiros, o fato de estarem formados e atuando a mais de 10 anos não teve relação com o diagnóstico de doenças crônicas, nem idade e tempo de diagnóstico e nem tempo de formação e diagnóstico, contrariando os achados do presente estudo. Entretanto, os resultados da mesma pesquisa se assemelharam com o perfil de enfermeiros com doenças crônicas quanto à carga horária semanal, crença espiritual e área de trabalho assistencial(11).

Sobre os resultados do presente estudo, destaca-se que se trata de trabalhadores em exercício profissional e que convivem com doenças crônicas, conforme indicam as variáveis associadas. Esse dado exige o repensar sobre as relações saúde-trabalho e, ainda, sobre o suporte oferecido aos enfermeiros que trabalham sob condições 
desfavoráveis à QV pela presença da doença, como a dor, o desconforto e os efeitos disso sobre a autoestima.

Cabe mencionar que a saúde do trabalhador é essencial para o desenvolvimento de qualquer instituição, seja do setor de saúde ou outro qualquer, contudo, o que se identifica é que cada vez mais as organizações cobram produtividade dos trabalhadores, sem oferecer condições favoráveis, para que estes possam desenvolver suas funções sem prejuízos à saúde ${ }^{(22)}$. Essa lógica neoliberal invade os serviços de saúde e também tem repercutido sobre a saúde dos enfermeiros, exigindo novos olhares sobre a saúde dos cuidadores de enfermagem.

\section{CONCLUSÕES}

A pesquisa realizada evidenciou uma parcela considerável de enfermeiros $(37,1 \%)$ que autoavaliaram sua saúde como razoável a ruim. Muitos deles convivem com doenças crônicas (31,2\%), como a hipertensão arterial, a diabetes mellitus, os transtornos depressivos e as doenças osteomusculares. Entende-se, portanto, que a associação das variáveis autoavaliação da saúde e doenças crônicas torna-se elemento importante para se analisar a QV destes profissionais. Cabe salientar que se trata de uma amostra que incluiu apenas trabalhadores em atividade, o que seria mais alarmante se incluídos os casos de afastamento.

No que se refere à descrição do perfil sociodemográfico dos enfermeiros, identificaram-se divergências em relação a outras pesquisas quanto à predominância de 133 (55,4\%) não casados, 128 (53,4\%) com um ou mais dependentes, 140 (58,3\%) atuantes no turno diurno, $133(55,4 \%)$ em até 40 horas semanais de trabalho e $203(84,6 \%)$ na assistência. Tem-se, portanto, um perfil distinto ao registrado na literatura, o que sinaliza as especificidades de cada contexto regional e aponta questões a serem consideradas no que tange à $Q V$ dos trabalhadores de enfermagem.

Os limites da investigação apresentada estão nos resultados regionalizados, que, em parte, corresponde à realidade geral no país, dos profissionais de enfermagem em seu ambiente de trabalho, com exceção da maioria dos enfermeiros, do município em questão, 183 (76,2\%) estar atuando em apenas um local de trabalho. Além disso, o instrumento utilizado é sucinto na abordagem da autoavaliação de saúde dos enfermeiros. Contudo, tratase de estudo relevante diante da possibilidade de servir de subsídio para a construção de estratégias que possam proporcionar melhores condições de vida e trabalho para os enfermeiros, e com isso, conseguir obter um nível adequado de QV no trabalho e, consequentemente, a prestação de uma assistência qualificada.

Assim, espera-se que esta pesquisa venha auxiliar a posteriores estudos, na medida em que reforça a necessidade de investimentos frente à problemática das doenças crônicas, bem como sensibiliza para as condições com que muitos enfermeiros trabalham, ou seja, sem por vezes considerar sua saúde como favorável. Diante disso, emerge a necessidade de se investir na QV dos enfermeiros, oferecendo melhores condições de trabalho e assistência à saúde dos trabalhadores.

\section{REFERÊNCIAS}

1. Fleck MPA. Grupo WHOQOL. Versão em português dos instrumentos de avaliação de qualidade de vida [Internet] 2010 [cited 2012 Mar 05]. Available from: http://www.ufrgs.br/psiq/whogol.html.

2. Marx K. O capital. Rio de Janeiro: Civilização Brasileira; 2004. 3. Ribeiro HP. Da Violência explicita à oculta. Ribeiro HP. In: A violência oculta no trabalho: as lesões por esforços repetitivos. Rio de Janeiro: Fiocruz; 1999. p. 37-50.

4. Estudo da saúde dos enfermeiros [Internet]. Fiocruz [cited 2012 Mar 05]. Available from:

http://www.ioc.fiocruz.br/enfsaude/saude.php.

5. Agostinho MR, Oliveira MC, Pinto MEB, Balardin GU, Harzheim E. Autopercepção da saúde entre usuários da Atenção Primária em Porto Alegre, RS. Rev. bras. Med. Fam. e Comun. 2010;5(17):9-15.

6. Díaz-Rodríguez L, Arroyo-Morales M, Cantarero-Villanueva I, Férnandez-Lao C, Polley M, Fernández-de-las-Peñas C. The application of Reiki in nurses diagnosed with Burnout Syndrome has beneficial effects on concentration of salivary IgA and blood pressure. Rev. latinoam enferm. 2011;19(5):1132-8.

7. Lacaz FAC, Vieira NP, Cortizo CT, Junqueira V, Santos APL, Santos FS. Quality of life, work management, and career planning as a primary care technology in the Unified National Health System in São Paulo State, Brazil. Cad. saúde pública. 2010;26(2):253-63.

8. Fernandes JS, Miranzi SSC, Iwamoto HH, Tavares DMS, Santos CB. Qualidade de vida dos enfermeiros das equipes de saúde da família: a relação das variáveis sociodemográficas. Texto \& contexto enferm. 2010;19(3):434-42.

9. Jylhä M. What is self-rated health and why does it predict mortality? Towards a unified conceptual model. Soc Sci Med. 2009;69(3):307-16.

10. Cavalheiro AM, Junior DFM, Lopes AC. Stress in nurses working in intensive care units. Rev. latinoam enferm. 2008;16(1):29-35.

11. Teixeira RC, Mantovani MF. Nurses with chronic illness: relations with the illness, prevention and the work process. Rev. Esc. Enferm. USP. 2009;43(2):415-21.

12. Barrientos LA, Suazo SV. Quality of life associated factors in Chileans hospitals nurses. Rev. latinoam enferm.

2007;15(3):480-6. 
13. Cárdenas AMC. Qualidade de vida de mulheres de uma comunidade de baixa renda: estudo comparativo entre mulheres com trabalho doméstico e trabalho remunerado [tese]. São Paulo (SP): Universidade de São Paulo. Escola de Enfermagem; 2004.

14. Trindade LL, Lautert L, Beck CLC, Amestoy SC, Pires DE. Stress and burnout syndrome among workers of the Family Health team. Acta paul. enferm. 2010;23(5):684-9.

15. Silva RM, Beck CLC, Guido LA, Lopes LFD, Santos JLG. Análise quantitativa da satisfação profissional dos enfermeiros que atuam no período noturno. Texto \& contexto enferm. 2009;18(2):298-305.

16. Santos I, Castro CB. Personal and professional nuances of nurses who perform administration functions in the university hospital. Rev. Esc. Enferm. USP. 2010;44(1):154-60.

17. Raffone AM, Hennington EA. Functional capacity evaluation of nursing professionals. Rev. saúde pública. 2005;39(4):669-76. 18. Calderero ARL, Miasso Al, Corradi-Webster CM. Estresse e estratégias de enfrentamento em uma equipe de enfermagem de Pronto Atendimento. Rev. eletrônica enferm. [Internet] 2008 [cited 2011 Nov 06];10(1):51-62. Available from:

http://www.fen.ufg.br/revista/v10/n1/pdf/v10n1a05.pdf. 19. Dias CB, Silva ALA. The profile and professional practice of nurses in a psychosocial care services. Rev. Esc. Enferm. USP. 2010;44(2):469-75.

20. Santos FD, Cunha MHF, Robazzi MLCC, Pedrao LJ, Silva LA, Terra FS. O estresse do enfermeiro nas unidades de terapia intensiva adulto: uma revisão da literatura. SMAD, SMAD, Rev. eletrônica saúde mental álcool drog. [Internet]. 2010 [cited 2011 Nov 05];6(1):1-16. Available from: http://pepsic.bvsalud.org/pdf/smad/v6n1/14.pdf.

21. Noronha APP, Fernandes DC. Estresse laboral: análise da produção científica brasileira no SciELO e BVS-Psi. Fractal rev. psicol. 2008;20(2):491-501.

22. Aguiar ADF, Barreto EO, Aguiar KS, Biazzini MG, Silva PM. Saúde do trabalhador de enfermagem que atua em centro de saúde. Rev. Inst. Invest. Cienc. Salud. 2009;27(2):103-8.

23. Murofuse NT, Marziale MHP. Diseases of the osteomuscular system in nursing workers. Rev. latinoam enferm.

2005;13(3):364-73.

24. Freitas JRS, Lunardi Filho WD, Lunardi VL, Freitas KSS. Distúrbios osteomusculares relacionados ao trabalho em profissionais de enfermagem de um hospital universitário. Rev. eletrônica enferm. [Internet]. 2009 [cited 2012 Fev

24];11(4):904-11. Available from:

http://www.fen.ufg.br/revista/v11/n4/pdf/v11n4a16.pdf. 25. Griep RH, Rotenberg L, Landsberg P, Vasconcellos-Silva PR. Combined use of job stress models and self-rated health in nursing. Rev. saúde pública. 2011;45(1):145-52.

Artigo recebido em 16/04/2012.

Aprovado para publicação em 24/01/2013.

Artigo publicado em 30/09/2013. 